\title{
Demeter high resolution observations of the ionospheric thermal plasma response to magnetospheric energy input during the magnetic storm of November 2004
}

\author{
E. Séran ${ }^{1}$, H. U. Frey ${ }^{2}$, M. Fillingim ${ }^{2}$, J.-J. Berthelier ${ }^{1}$, R. Pottelette ${ }^{1}$, and G. Parks ${ }^{2}$ \\ ${ }^{1}$ CETP, 4 Avenue de Neptune, 94100 Saint-Maur, France \\ ${ }^{2}$ SSL, University of California, Berkeley, USA
}

Received: 24 January 2007 - Revised: 25 October 2007 - Accepted: 14 November 2007 - Published: 2 January 2008

\begin{abstract}
High resolution Demeter plasma and wave observations were available during one of the geomagnetic storms of November 2004 when the ionospheric footprint of the plasmasphere was pushed below 64 degrees in the midnight sector. We report here onboard observations of thermal/suprathermal plasma and HF electric field variations with a temporal resolution of $0.4 \mathrm{~s}$, which corresponds to a spatial resolution of $3 \mathrm{~km}$. Local perturbations of the plasma parameters at the altitude of $730 \mathrm{~km}$ are analysed with respect to the variation of the field-aligned currents, electron and proton precipitation and large-scale electric fields, measured in-situ by Demeter and by remote optical methods from the IMAGE/Polar satellites.
\end{abstract}

Flow monitoring in the 21:00 and 24:00 MLT sectors during storm conditions reveals two distinct regions of $\mathrm{O}^{+}$outflow, i.e. the region of the field-aligned currents, which often comprises few layers of opposite currents, and the region of velocity reversal toward dusk at sub-auroral latitudes. Average upward $\mathrm{O}^{+}$velocities are identical in both local time sectors and vary between 200 and $450 \mathrm{~m} \mathrm{~s}^{-1}$, with an exception of a few cases of higher speed $\left(\sim 1000 \mathrm{~m} \mathrm{~s}^{-1}\right)$ outflow, observed in the midnight sector. Each individual outflow event does not indicate any heating process of the thermal $\mathrm{O}^{+}$population. On the contrary, the temperature of the $\mathrm{O}^{+}$, outflowing from auroral latitudes, is found to be even colder than that of the ambient ion plasma. The only ion population which is observed to be involved in the heating is the $\mathrm{O}^{+}$with energies a few times higher than the thermal energy. Such a population was detected at sub-auroral latitudes in the region of duskward flow reversal. Its temperature raises up to a few $\mathrm{eV}$ inside the layer of sheared velocity.

A deep decrease in the $\mathrm{H}^{+}$density at heights and latitudes, where, according to the IRI model, these ions are expected to comprise $\sim 50 \%$ of the positive charge, indicates that the

Correspondence to: E. Séran

(seran@ cetp.ipsl.fr) thermospheric balance between atomic oxygen and hydrogen was re-established in favour of oxygen. As a consequence, the charge exchange between oxygen and hydrogen does not effectively limit the $\mathrm{O}^{+}$production in the regions of the electron precipitation. According to Demeter observations, the $\mathrm{O}^{+}$concentration is doubled inside the layers with upward currents (downward electrons). Such a density excess creates the pressure gradient which drives the plasma away from the overdense regions, i.e. first, from the layers of precipitating electrons and then upward along the layers of downward current.

In addition, the downward currents are identified to be the source regions of hiss emissions, i.e. electron acoustic mode excited via the Landau resonance in the multi-component electron plasma. Such instabilities, which are often observed in the auroral region at 2-5 Earth radii, but rarely at ionospheric altitudes, are believed to be generated by an electron beam which moves through the background plasma with a velocity higher than its thermal velocity.

Keywords. Ionosphere (Auroral ionosphere, Ionospheremagnetosphere interactions, Particle precipitation)

\section{Introduction}

The main interest of this paper is the plasma modification in the auroral and sub-auroral regions of the Earth nightside ionosphere during a strong magnetic storm. Tail stretching and associated processes, like magnetic reconnection, current sheet instabilities, etc., which develop in the tail due to magnetic storms, have a direct impact on the nightside ionosphere (Hultqvist et al., 1999). The energy, which is carried by intense, large-scale electric fields and also by energetic particles, is transformed at ionospheric altitudes into plasma acceleration, heating, ionisation of the neutral population, photoemissions, waves, etc. This transformation, which is

Published by Copernicus Publications on behalf of the European Geosciences Union. 
height and time dependent, modifies the global balance of the ionosphere. Part of the energy is carried back to the magnetosphere by the charged particles that escape from the Earth's gravity due to their energization along the magnetic field lines. The ionospheric plasma is estimated to be a dominant source of the near-Earth magnetospheric plasma. The ion outflow from the ionosphere is strongly correlated (Yau et al., 1988) with the solar and magnetic activity. Total outflow from both hemispheres reaches $\sim 10^{26}$ ions s $^{-1}$ during magnetic storms. A comprehensive review of a large variety of physical processes which are responsible for the plasma exchange between the ionosphere and magnetosphere is presented in the book by Hultqvist et al. (1999) and the paper by André and Yau, (1997). However, some ambiguities remain in the mechanisms of the energization of thermal ionospheric plasma and its transport into the magnetosphere.

A few attempts were made to correlate the ion outflow with the precipitation of the auroral electrons and of the ring current heavy ions. Yamamoto et al. (1993), Hirahara et al. (1998) concluded that the ion beams observed above $5000 \mathrm{~km}$ are often associated with bright, discrete auroral signatures, contrary to the ion conics which were often seen outside the regions of intense electron precipitation or not even allied to any UV emission (Wilson et al., 2001). Wahlund and Opgenoorth (1989) emphasized that intense ion outflows are not correlated with an ion temperature enhancement, but rather with an increased ion production and electron heating. At sub-auroral latitudes, the enhanced $\mathrm{O}^{+}$production and related upward ion acceleration at the heights between 600 and $800 \mathrm{~km}$ are suggested (Yeh and Foster, 1990; Torr et al., 1974) to be associated with the ring current energization. According to Torr et al. (1974), the intense ring current $\mathrm{O}^{+}$ions, which precipitate into the ionosphere, can produce large upward fluxes of energetic atomic oxygen in the top ionosphere. While moving upward these atoms are ionised through collisions and transfer their energy to $\mathrm{O}^{+}$ ions.

In the present paper, we analyse the plasma disturbances observed in the regions of auroral precipitation and subauroral polarisation stream (SAPS). In the literature a westward reversal of plasma flow at sub-auroral latitudes is referenced as "polarisation jets" (Galperin, 1973; Yeh and Foster, 1990) or Subauroral Ion Drift (SAID) (Southwood and Wolf, 1978; Anderson et al., 2001) or Subauroral Polarisation Stream (SAPS) (Oksavik et al., 2006). Such streams are formed during magnetically disturbed conditions, have the latitudinal width of a few degrees, a speed of about $500 \mathrm{~m} \mathrm{~s}^{-1}$ and are suggested to be driven by the polarization electric field created at the outer boundary of the ring current due to a different depth of injection of the plasma sheet ions and electrons. The polarisation field mapped along the geomagnetic field lines to the subauroral ionosphere has a poleward direction and therefore drives duskward plasma convection.

We discuss the current structuring associated with the large-scale electric field modulations, the variation of the $\mathrm{O}^{+}$ density correlated with the intensity of the precipitating electrons, upward electron acceleration and generated hiss, escape of the $\mathrm{H}^{+}$from the top ionosphere and outflowing of the cold $\mathrm{O}^{+}$population, plasma acceleration and heating of the suprathermal ions in the sub-auroral regions.

We start in Sect. 2 with a brief description of the onboard instruments. An overview of the magnetospheric and then ionospheric modifications during the exceptionally long period of the magnetic storm is made in Sect. 3.1. Thorough analysis of the ionospheric plasma response on the energy input during the substorm development is performed in Sect. 3.2. The main results are summarised in the Conclusions.

\section{Instrumentation}

In this paper we use the observations made by the UV cameras on board the IMAGE and Polar satellites and by the plasma instruments on board the Demeter satellite. The advantage of such a data set is that it provides quasisimultaneous information about the energy input into the ionosphere and resulted plasma perturbations at the different ionospheric layers, i.e. at the heights of $\sim 150 \mathrm{~km}$ (UV imagers) and $730 \mathrm{~km}$ (Demeter).

Onboard imagers are the optical cameras (Torr et al., 1995; Mende et al., 2000) that detect the photoemissions in the ultra-violet frequency range. The IMAGE WIC and Polar LBHS are centred at $150 \mathrm{~nm}$ and the Polar LBHL at $170 \mathrm{~nm}$. The Polar imagers both have about a $20 \mathrm{~nm}$ bandpass. The bandpass for the IMAGE FUV WIC is much larger, 40 to $50 \mathrm{~nm}$. All these cameras detect the emissions of $\mathrm{N}_{2}$ due to the impact of keV electrons. The Spectrographic Imager (SI/Image) is sensitive to the Doppler shifted Lyman H-alpha line emissions centred at $121.82 \mathrm{~nm}$ that is generated by energetic protons.

The Demeter onboard instruments, the measurements of which are used in the present paper, are retarding (APR) and directional (ADV) ion analysers, electric field antenna (ICE) and fluxgate magnetometer.

The APR retarding analyser is designed to measure atomic and molecular ions with densities down to a few particles per $\mathrm{cm}^{-3}$ and relative masses up to $\sim 56$. The entrance of the charged species into the analyser is controlled by the polarisation grids. One of them is polarised at $-12 \mathrm{~V}$ and does not allow the electrons with energies lower than $12 \mathrm{eV}$ to reach the collector. The potential applied to another grid is positive and gradually varies between 0 and $\sim 20 \mathrm{~V}$ during each sequence of measurements. The variation of the current collected by the analyser versus the grid potential is used to deduce the ion plasma composition, the concentration of each species, the temperature and velocity parallel to the analyser axis.

The ADV, like APR, measures the current due to positive ions. But the grid potentials are fixed and the 


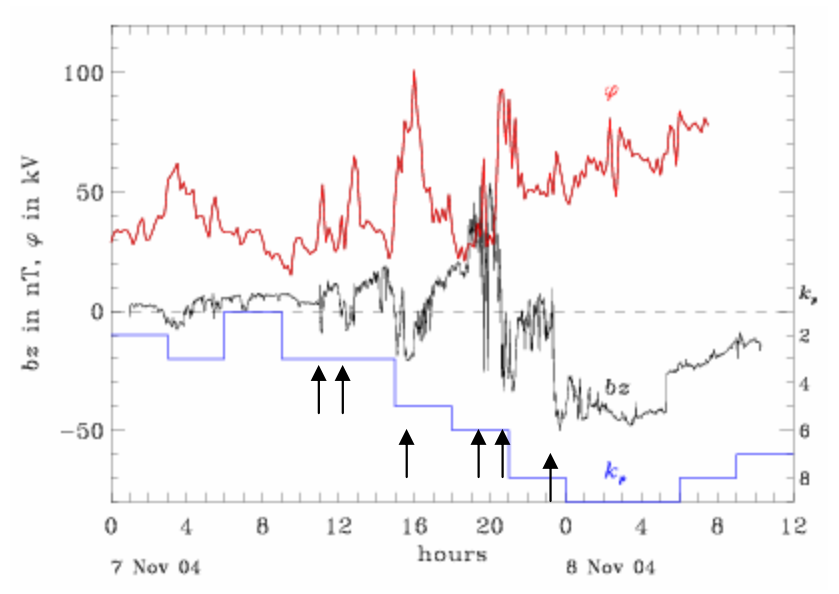

Fig. 1. Variation of (i) transpolar potential deduced from the SuperDARN flow observations in the ionosphere, (ii) $K_{p}$ index calculated from the ground-based magnetic records and (iii) south-north component, $B_{z}$, of the interplanetary magnetic field measured on board the ACE satellite on 7 and 8 November 2004. Arrows indicate the southward rotation of the interplanetary magnetic field. Magnetic records in the solar wind are shifted by $1 \mathrm{~h}$.

collector comprises four identical parts. Thus, the combination of currents measured by each sub-collector allows one to estimate the component of flow velocity in the plane perpendicular to the analyser axis (it points along the direction of the satellite motion). A detailed description of both instruments and models that are used to deduce the plasma parameters can be found in the papers by Séran (2003) and Berthelier et al. (2006a).

The electric field antenna (ICE) comprises four spherical monopoles. A combination of dc/ac potentials measured by each sphere allows one to deduce the electric field variations up to the frequency of 3.3 MHz (Berthelier et al., 2006b).

For overview purposes we use magnetic observations in the solar wind by the ACE satellite at the Lagrangian point L1, the ionospheric flow survey by the polar ground-based network of the SuperDARN HF radars and the magnetic records by sub-auroral ground-based magnetometers.

\section{Observations and discussion}

3.1 Magnetospheric/ionospheric response to the magnetic storm

Observations which are presented in this paper were made on 7 and 8 November 2004 during a huge magnetic storm which commenced at $\sim 10: 00$ UT on 7 November and developed over $\sim 28 \mathrm{~h}$. The magnetic induction in solar wind measured on board the ACE spacecraft reached $50 \mathrm{nT}$ and stayed at this level during almost $10 \mathrm{~h}$. Under such conditions one can expect an intense mass-loading of the solar wind plasma and consequent plasma transport between
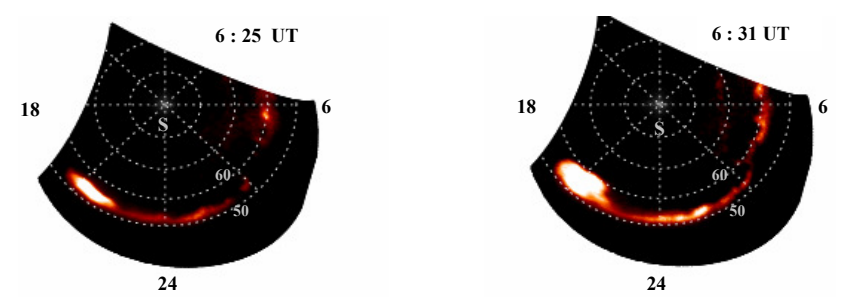

Fig. 2. Two consecutive WIC images made in the night sector of the Southern Hemisphere on 8 November. Distribution of the emission intensity is represented in MLT/magnetic latitude coordinates.

ionosphere and magnetosphere. In order to give an overview of the magnetospheric response during the storm development, we present in Fig. 1 the variations of (i) the transpolar potential, $\varphi$, deduced from the $E x B$ flows measured by the SuperDARN coherent radars in the polar and auroral ionosphere of the Northern Hemisphere, and of (ii) the $K_{p}$ index, calculated as the mean value of the horizontal magnetic field disturbances, recorded by ground-based magnetometers, together with (iii) the variation of the south-north component, $B_{z}$, of the interplanetary magnetic field, monitored by the ACE spacecraft at the L1 point. For the purposes of comparison, the last parameter is shifted by 1 hour, which is approximately the propagation time of the solar wind plasma from ACE to Earth. Good correlation between the solar wind dynamics and the magnetospheric response is clearly seen from the presented plots. Each rotation of the interplanetary magnetic field toward south $\left(B_{z}<0\right)$ results in an enhancement of the transpolar potential, the amplitude of which is mainly determined by the duration of the magnetic field southward orientation. The level of magnetospheric activity represented by $K_{p}$ follows the variation of the magnetic pressure in the solar wind whenever $B_{z}<0$. The $K_{p}$ index reaches extremely high levels, i.e. 8-9, at the end of 7 November, and stays high for $\sim 12 \mathrm{~h}$. This results in the polar cap expansion and associated displacement of the auroral oval to lower latitudes. The UV images recorded by the WIC camera on board the IMAGE satellite at $\sim 06: 30$ UT perfectly illustrate this extension (Fig. 2). The emissions that indicate the auroral oval position are localised below $60^{\circ}$ magnetic latitude in the nightside sector. During the whole period of storm development, the ADV analyser on board Demeter detects the flow reversal towards the dusk which occurs at sub-auroral latitudes in both 21:00 and 24:00 MLT sectors of Northern and Southern Hemispheres. SAPS boundaries are observed to displace toward the equator by almost $15^{\circ}$ (see Fig. 3). This motion is directly related to the storm intensification (i.e. $K_{p}$ surpasses 7).

Another component deduced from the ADV measurements is the vertical velocity. Bursts of the upgoing $\mathrm{O}^{+}$(here we call "burst" a vertical ion motion with the mean velocity higher than $200 \mathrm{~m} \mathrm{~s}^{-1}$ ) were observed over the large latitudinal range, i.e. from $30^{\circ}$ to $60^{\circ}$. The latitudinal distribution 


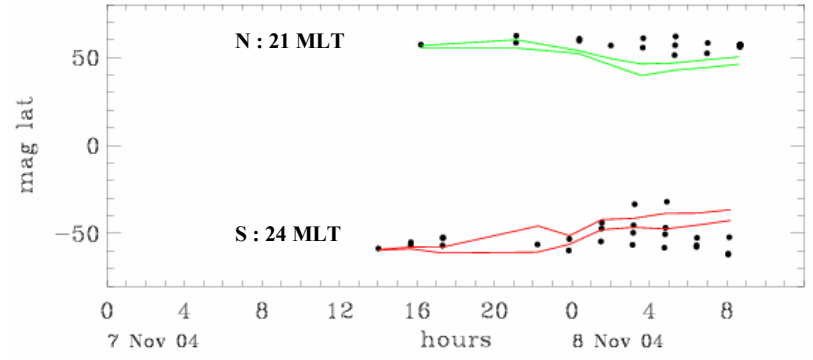

Fig. 3. Temporal variation of the latitudes which correspond to the boundaries of duskward flows, as deduced from the ADV measurements at 21:00 and 24:00 MLT sectors of the Northern (green line) and Southern (red line) Hemispheres, respectively. Dots indicate the latitudes of the observed $\mathrm{O}^{+}$outflow with the mean velocity higher than $200 \mathrm{~m} \mathrm{~s}^{-1}$. The observations were made at the latitudes below magnetic $62^{\circ}$.

of such events versus time is represented in Fig. 3 by dots. The difference between their locations with respect to the SAPS boundaries in two local time sectors is remarkable. In contrast to the broad extension of outflow events over auroral/sub-auroral latitudes at the midnight sector, the midevening outflow is limited to higher latitudes. We see at least two likely reasons for the outflow observations at the subauroral regions, i.e. (i) duskward electric field, which drives the upgoing $\mathrm{O}^{+}$equatorward from the auroral source regions, or/and (ii) sub-auroral source of the $\mathrm{O}^{+}$outflowing. The average vertical speed of the detected $\mathrm{O}^{+}$bursts is estimated to vary between 200 and $450 \mathrm{~m} \mathrm{~s}^{-1}$ in both magnetic local time sectors, i.e. 21:00 MLT and 24:00 MLT, with the exception of a few events with higher speed, i.e. $\sim 1000 \mathrm{~m} \mathrm{~s}^{-1}$. Each of the high-speed events is mapped to the region of the fieldaligned currents and corresponds to currents with densities of 5-9 $\mu \mathrm{A} \mathrm{m}^{-2}$, i.e. about 2 times higher than in the majority of observed events.

\subsection{Midnight observations during the conditions with $K_{p}=8$}

In order to place the observations in a global context, let us come back to Fig. 2, which shows two consecutive WIC images made at 06:25 and 06:31 UT on 8 November. The images, which cover almost entirely the high latitudes of the night part of the Southern Hemisphere, illustrate a growth phase of sub-storm development. A narrow spot of precipitating electrons, which appears in the first image between 21:00 and 22:00 MLT, expands then towards the pole and dusk. This expansion is accompanied by an intensification of the emission in the post-midnight sector. The relative intensity of the emissions with two wavelenght, i.e. 150 and $170 \mathrm{~nm}$, made by the UVI camera on board the Polar satellite, which covered the same region as the Image cameras and made its observations at the same time, allows to estimate (using the method developed by Germany et al., 1998) the associated energies of the precipitating electrons. We found 9 and $5 \mathrm{keV}$ in the 21:00 and 24:00 MLT sectors, respectively. During this time Demeter moves from higher latitudes toward the equator and makes the observations in the magnetic sector between 00:02 and 00:22 MLT, i.e. it crosses the part of the auroral oval which is typically associated with the downward currents that connect the tail and the ionosphere. In order to compare the two data sets, the latitudinal distribution of the emissions detected by the WIC and SI12 cameras inside the magnetic local sector of the Demeter path are drawn in Figs. $4 \mathrm{a}$ and $4 \mathrm{~b}$, respectively, together with the density distribution of the field-aligned currents deduced from the magnetic perturbations measured on board Demeter (blue line). The currents of both polarities (upward and downward, represented, respectively, by the negative and positive density) co-exist and have approximately the same magnitude, i.e. 2-3 $\mu \mathrm{A} \mathrm{m}^{-2}$. The $\mathrm{H}$-line emission (red line in Fig. $4 \mathrm{~b}$ ) closely follows the latitudinal distribution of the field-aligned current, i.e. the emission intensity is increased inside the layers of downward current and decreased in the regions of upward current. These observations give evidence that the precipitating protons, which produce the emissions, follow the currents that circulate between the tail and the ionosphere and probably contribute to them. The $\mathrm{N}_{2}$-emissions (red line in Fig. 4a) encompass the magnetic latitudes between $-56^{\circ}$ and $-48^{\circ}$. With respect to these emissions the field-aligned currents are observed in the broader region extending toward the pole. We note that the emission intensification does not exactly correlate with the upward current distribution. Such a discrepancy can have different reasons. We just mention a few of them, i.e.

- field-perpendicular diffusion of the energetic electrons due to collisions in the lower ionosphere;

- integration of emissions over the large interaction layer along the line of sight which significantly deviates from the local magnetic field direction;

- spatial and/or temporal variations in the distribution of thermospheric/ionospheric populations.

Under similar conditions Peterson et al. (1994) have shown that there is a significant increase in molecular ions, such as $\mathrm{NO}^{+}, \mathrm{O}_{2}^{+}$and $\mathrm{N}_{2}^{+}$. Unfortunately, the APR analyser on board Demeter can not distinguish these species in the auroral regions which are populated with suprathermal electrons. Designed to measure the ion (positive) currents the analyser is protected by a negatively polarised grid from the electron collection. However, the grid potential, which is fixed at $-12 \mathrm{~V}$, is not sufficient to stop the electrons with energies higher than $12 \mathrm{eV}$. This results in the annulation of the positive current produced by minor positively charged species whenever the flow of suprathermal electrons exceeds the flow of the minor ions. For example, in the considered case the 

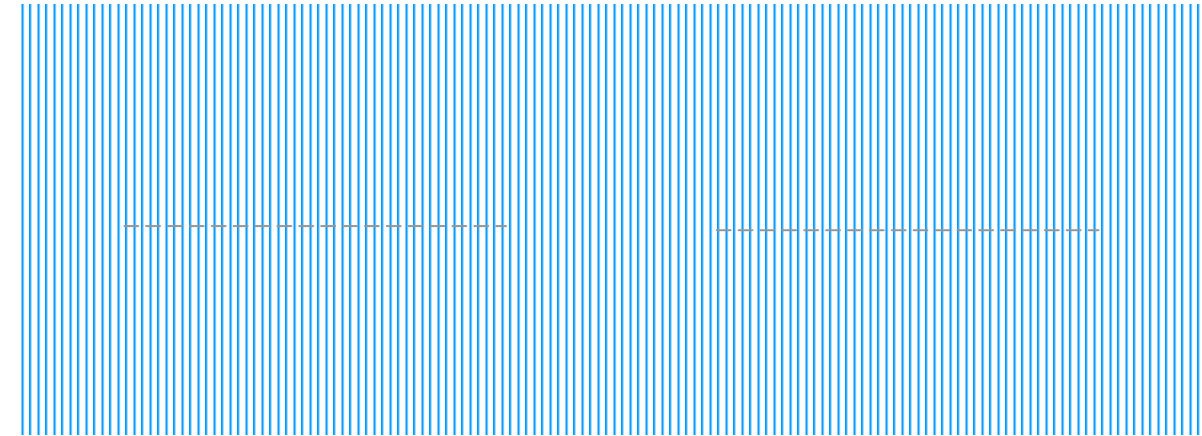

Fig. 4. Latitudinal distribution of the emission detected by the (a) WIC and (b) SI12 cameras (both are shown in red), together with the fieldaligned current deduced from the magnetic perturbations measured on board the Demeter (in blue). Upward and downward are represented, respectively, by the negative and positive current density. Both observations are made along the magnetic local sector comprised between 00:02 and 00:22 MLT, which is covered by Demeter when it moves from $-55^{\circ}$ to $-48^{\circ}$ magnetic latitude.

suprathermal electrons are estimated to comprise up to $20 \%$ of the total density. Therefore, the ions with density below $4000 \mathrm{~cm}^{-3}$ are not resolved.

Contrary to a basic model of the current system, which predicts a couple of anti-parallel field-aligned currents flowing between the tail and ionosphere in the midnight sector, with the downward current poleward with respect to the upward current, several current layers are observed in the region between the $-62^{\circ}$ and $-48^{\circ}$ magnetic latitude. Each layer with the width of $\sim 400 \mathrm{~km}$ comprises a pair of anti-parallel currents, i.e. downward poleward and upward equatorward. Such multi-structuring observed at the ionospheric heights most likely reflects the spatial configuration of the source region or/and its temporal evolution.

How does the ionosphere respond to the energy/mass input? With the intention to find a response, let us analyse the variation of the thermal ion parameters inside the auroral and sub-auroral regions, taking advantage of Demeter observations.

\subsubsection{Ion plasma convection}

Two components of the ion velocity, i.e. horizontal (in red) and vertical (in blue), measured by the ADV analyser in the plane perpendicular to the satellite orbital motion, are presented in Fig. 5. The velocities are positive/negative when the ions move dawn/dusk and down/up, respectively. At the latitudes poleward of $-45^{\circ}$, the ionospheric plasma flows dawnward. This motion is associated with the plasma convection across the polar cap driven by the solar wind and subsequent plasma return along the magnetospheric boundaries. The observed component of the convection speed (red line) stays high, i.e. about $1000 \mathrm{~m} \mathrm{~s}^{-1}$, over the whole region that encompasses the field-aligned currents. This convection is associated with the equatorward electric field of $\sim 40 \mathrm{mV} \mathrm{m}^{-1}$. The large-scale changes in the azimuthal velocity with amplitude of $\sim 200 \mathrm{~m} \mathrm{~s}^{-1}$ correlate with the current variation (green line in the same figure). Note that the

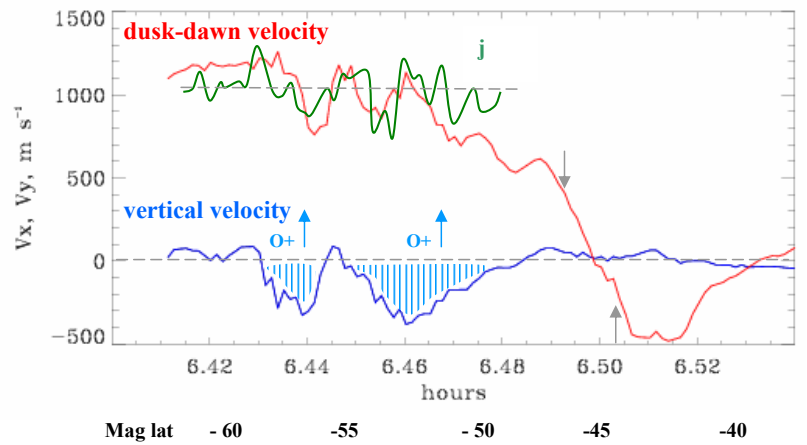

Fig. 5. Two components of the ion plasma velocity observed at $730 \mathrm{~km}$ by ADV when Demeter crosses the auroral oval. Red and blue stand for the horizontal and vertical velocities, respectively, measured in the plane perpendicular to the satellite orbital motion. Velocities are positive/negative when plasma moves toward dawn/dusk and downward/upward.

maximum current intensity, in general, corresponds to the maximum velocity gradient, but the magnetic induction, $|B|$, does not. Thus, these waves are non-compressional and are presumably driven by the field-aligned currents.

The flow reversal toward dusk is observed at latitudes equatorward of $-45^{\circ}$ and has the latitudinal width of about $800 \mathrm{~km}$ with a high speed channel which extends over $250 \mathrm{~km}$. Observed duskward flows are suggested to be driven by the polarisation electric field established at the outer edge of the ring current and caused by a deeper injection of the plasma sheet ions into the ring current with respect to the electrons. Thus, the typical scale of such charge separation is of the order of $\mathrm{O}^{+}$gyrodiameter, i.e. about $500-1000 \mathrm{~km}$. Mapped to the ionospheric altitudes this field has a poleward direction, which is opposite to that which drives the post midnight convection. 

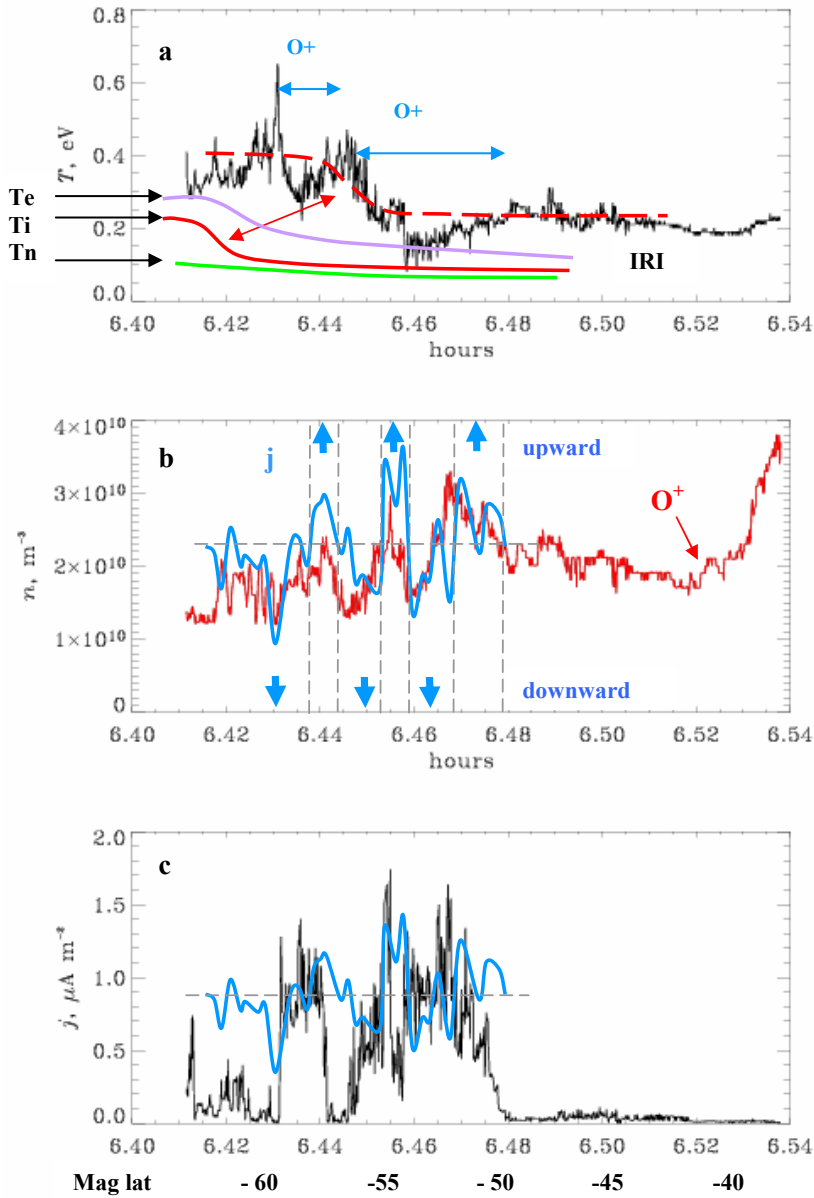

Fig. 6. Plasma parameters measured by the APR analyser during the same period as in Fig. 5, i.e. (a) $\mathrm{O}^{+}$temperature (in black), together with the electron (in violet), ion (in red) and neutral (in green) temperatures deduced from the IRI model; (b) $\mathrm{O}^{+}$density (in red), together with the current density (in blue), as deduced from the magnetic perturbations; (c) current density due to the electrons with energy higher than $12 \mathrm{eV}$. Regions of $\mathrm{O}^{+}$outflow are indicated by horizontal arrows. The layers of anti-parallel currents are highlighted by vertical dashed lines and the current direction is indicated by arrows.

\subsubsection{Thermal balance}

Presented in Fig. 6a is the $\mathrm{O}^{+}$temperature deduced from the APR data, together with ion, electron and neutral temperatures given by the IRI model. The unusually high (with respect to the model prediction) average $\mathrm{O}^{+}$temperature is most likely caused by the unusually long and intense period of the magnetic storm. We can cite few mechanisms which might contribute to the heating of the nightside high-latitude ionosphere, i.e.

- electron precipitation with consecutive heating of thermal electrons in the lower ionosphere;
- frictional heating in the regions of strong convection;

- heat convection from the day-to nightside ionosphere, driven by the plasma convection across the polar cap;

- solar wind entry along the open field lines.

But there are other sources which also contribute to the thermal state of the ionosphere. In the example shown, significant temperature variations are observed in the regions that envelope the layers of the field-aligned currents. A deep temperature decrease around $\sim 6.435$ and 6.46 corresponds to the moments of the $\mathrm{O}^{+}$outflowing. At the Demeter heights the thermal exchange between the outflowing and ambient plasma will likely result in a cooling of local plasma. Two thermal regimes are clearly distinguished from both curves presented results of modelling and observations. The ion temperature is significantly higher in the regions of the polar cap and jumps down across the current layers.

A remarkable feature observed at the sub-auroral latitudes and associated with the SAPS boundaries is the heating of the suprathermal population. Typical observations of such heating are shown in Fig. 7a, b. Distribution of the current collected by the APR detector versus potential applied to the retarding grid illustrates the instrument response in the plasma with two co-existent $\mathrm{O}^{+}$species, i.e. cold with a temperature of fractions of $\mathrm{eV}$ and warm with a temperature of a few $\mathrm{eV}$. The fact that the heated $\mathrm{O}^{+}$consists of $\sim 2 \%$ (i.e. $\sim 400 \mathrm{~cm}^{-3}$ ) thermal ions and that the main part of the distribution function stays at the same temperature demonstrates that only suprathermal particles with thermal velocities higher than $2 V_{T}$ are involved in the heating process. Two examples presented in Fig. 7 correspond to the moments indicated by the arrows in Fig. 5, i.e. outside a and inside $b$ the SAPS convection. Suprathermal heating commences at about 6.49 , i.e. at the edge of the layer with strong sheared velocity. The temperature of suprathermal $\mathrm{O}^{+}$is gradually amplified and reaches $\sim 5 \mathrm{eV}$. Of course, the observed $5 \mathrm{eV}$ is a lower limit of energy required to overcome the Earth's gravity. Nevertheless, this example indicates a potential source of the $\mathrm{O}^{+}$outflowing, i.e. heating associated with the flow reversal at the sub-auroral latitudes.

\subsubsection{Plasma composition and density}

According to the IRI model, two major positive species, i.e. $\mathrm{O}^{+}$and $\mathrm{H}^{+}$, are expected to populate the considered latitudes and heights of the mid-night ionosphere. The density of $\mathrm{O}^{+}$, which is the dominant ion below $700 \mathrm{~km}$, drops down with height and the charge neutrality at the higher altitudes is mainly maintained by the $\mathrm{H}^{+}$species. At $700 \mathrm{~km}$ the $\mathrm{H}^{+}$ is expected to comprise $\sim 50 \%$ of the positive charge. However, this ion is not distinguished in the APR measurements and therefore its density does not exceed $\sim 1 \%$ of $\mathrm{O}^{+}$. Possible displacement of the $\mathrm{H}^{+}$at the higher altitudes will result in the re-establishment of the ionospheric height scales 

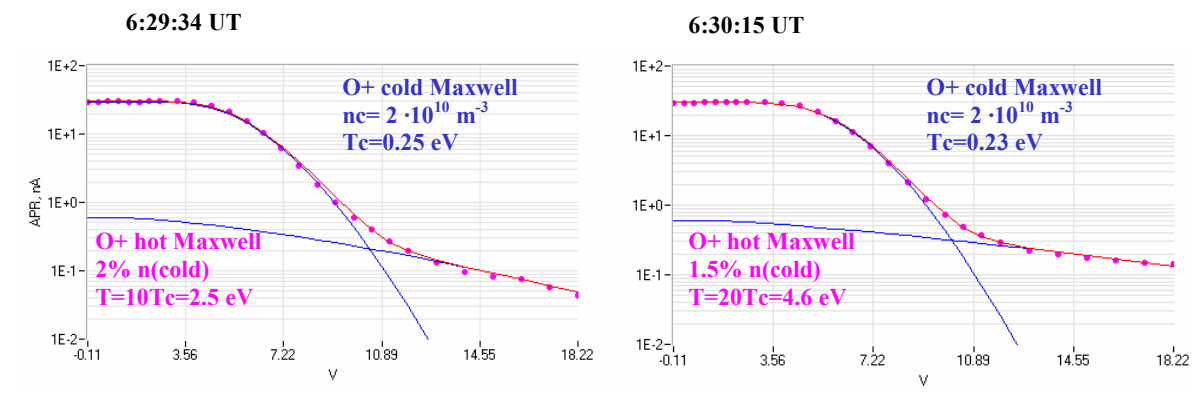

Fig. 7. Current distribution versus potential applied to the retarding grid measured by APR shows two populations of the $\mathrm{O}^{+}$species, i.e. cold and warm. Moments of observations are indicated by arrows in Fig. 5. Temperature of the warm population is gradually increased inside the SAPS boundary layer.

and thermospheric balance between oxygen and hydrogen. In such a situation the charge exchange between $\mathrm{O}^{+}$and hydrogen does not effectively limit the $\mathrm{O}^{+}$production in the regions of the electron precipitation. Thus, one can expect an increase in the $\mathrm{O}^{+}$concentration in the layers of upward current. Indeed, shown in Fig. $6 \mathrm{~b}$, the variation of the $\mathrm{O}^{+}$ density (in red) together with the field-aligned current density (in blue) confirms this conclusion. In order to make a straightforward comparison of the two plots, the sign of the current was changed to the opposite with respect to that in Figs. 4 and 5. Thus, a positive value means an upward current.

As explained before, the APR analyser, which was designed to measure positively charged species, is not protected from the collection of suprathermal electrons with energies higher than $12 \mathrm{eV}$. In the auroral regions, where this population is significant, the high energy electrons that reach the analyser create the negative current which compensates the current carried by the minor ion populations. The negative current consists of up to $20 \%$ of the positive ion current. Plotting the electron current densities deduced from the APR data (black line in Fig. 6c) and those estimated from the magnetometer measurements (blue line), we note a similarity in their variations. Each enhancement of the electron current deduced from the retarding analyser is associated with the upward current seen by the magnetometer. Observed differences in amplitude and variations could be caused by at least two reasons. First, the magnetic perturbations which are used to deduce the total current are not necessarily induced uniquely by the crossed current structure, but reflect an ensemble of the nearby currents. In contrast, the negative current collected by the ion analyser is due to the local electron flow that reaches the collector. Second, the angle between the analyser axis and the magnetic field varies between 85 to $75^{\circ}$ during the considered period. Having an acceptance angle of $106^{\circ}$ the analyser will collect the electrons with a velocity vector which consists of an angle larger than $22^{\circ}$ with respect to the magnetic field direction. Therefore, the collected negative current contains two components,

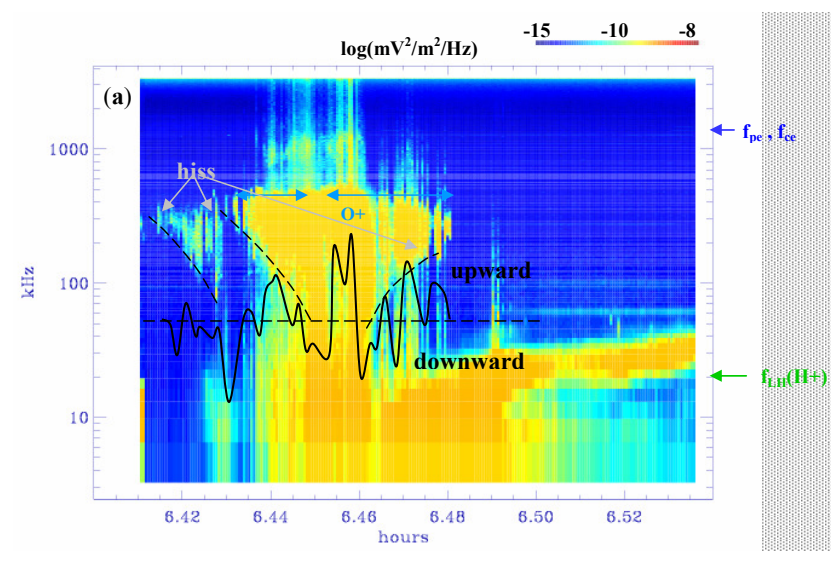

Fig. 8. HF electric field variations in $(30 \mathrm{kHz}, 3.3 \mathrm{MHz})$ frequency range during the same period as in Figs. 5, 6. Funnel-shaped frequency time structures associated with hiss emissions are indicated by dashed lines. Solid line stands for the field-aligned current density; positive for the upward and negative for downward current direction. Regions of $\mathrm{O}^{+}$outflow are indicated by horizontal arrows.

i.e. the first is due to the field-aligned electron flow and the second is due to the field-perpendicular motion. The second component does not contribute to the total current, since in the case of an isotropic field perpendicular particle distribution it is compensated by the opposite electron motion.

\subsection{4 $\mathrm{O}^{+}$outgoing}

The auroral region is characterised not only by the fieldaligned motion of light charged particles, like electrons and protons, but also by heavier ions, like $\mathrm{O}^{+}$. Indicated as a hatched area in Fig. 5, the outflowing $\mathrm{O}^{+}$is observed inside the region of the field-aligned currents, has the upward speed of $300 \mathrm{~m} \mathrm{~s}^{-1}$ and is essentially colder than the surrounding ion plasma (see Fig. 6a and previous discussion). What is the source of this motion? Detailed analysis of the plasma dynamics given before, is in favour of outflowing caused by the plasma pressure gradients inside the current region, with 


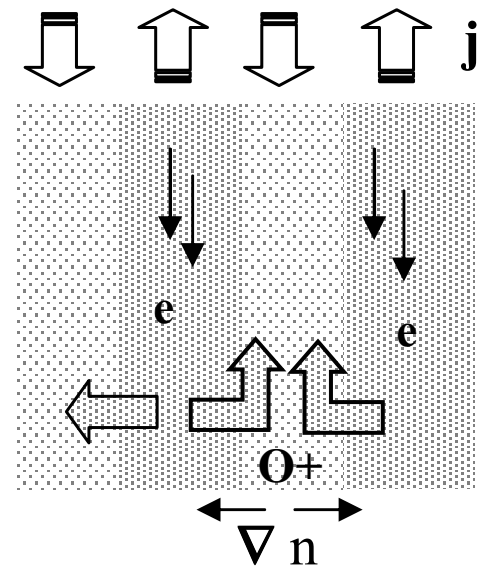

Fig. 9. A schematic drawing which illustrates two pairs of antiparallel currents, enhanced $\mathrm{O}^{+}$production inside the regions of the electron precipitation and $\mathrm{O}^{+}$outflowing from the overdense layers.

few a layers of opposite currents. The $\mathrm{O}^{+}$, driven away from the overdense layers of upward current, is then confined in the layer of downward current and pushed upward. The fact that in spite of a strong convection, the upgoing $\mathrm{O}^{+}$observed in the regions of the field-aligned currents indicates that the plasma mainly drifts along the current layers and/or the upward acceleration takes place not far from the heights of the Demeter observations (Yeh and Foster, 1990).

\subsubsection{Waves}

Supplementary information about the plasma processes developed in the auroral region can be taken from the HF electric field measurements. Remarkable wide-band emissions with the upper cutoff at $500 \mathrm{kHz}$ are observed (Fig. 8) in the moments when the satellite crosses the current layers. The excited frequencies lie well below the local electron cyclotron and plasma frequencies and well above the ions frequencies. The spectral width of the emissions is about $100 \mathrm{kHz}$ at the flanks of the current region and grows up to $400 \mathrm{kHz}$ in the vicinity of layers with downward current. Such waves have the properties similar to those observed in the auroral regions at $2-5 \mathrm{R}_{E}$, referred to as hiss (see, for example, Gurnett et al., 1983; Lin et al., 1984), and are believed to be generated locally by the upgoing electron beams. The distinctive funnel-shaped frequency time structure of hiss is explained (Lin et al., 1984) as a propagating effect, i.e. the group velocity of the low frequency waves points along the magnetic field lines and of the high frequency waves is directed at larger angles with respect to the field lines associated with the beam motion. A large spectral width of the observed emissions is probably due to the large source extension along the field lines. In the multi-component electron plasma the electron acoustic instability is excited via the Landau resonance and has the upper frequency cutoff which approximately corresponds to the plasma frequency of the secondary electron population (Tokar and Gary, 1984). The observed upper frequency cutoff of $500 \mathrm{kHz}$ gives the density estimate of $\sim 3 \times 10^{3} \mathrm{~cm}^{-3}$, which represents $\sim 15 \%$ of the local plasma density at $700 \mathrm{~km}$. The growth of the hiss mode requires that the resonance velocities lie in the region with a sufficiently positive derivative of the distribution function, i.e. above the thermal electron speed.

\section{Conclusions}

The principal purpose of this paper is to take advantage of simultaneous IMAGE-Polar-Demeter observations and to perform a detailed analysis of the ionospheric modifications due to intense energy/mass exchange during substorm development. Remote observations made by the UV cameras on board IMAGE and Polar give quasi-instantaneous images of photoemissions produced by the collisions and charge exchanges between energetic particles of magnetospheric origin and the species that populate the low thermosphere. Even if the emission intensities are highly representative, since they carry indirect information related to the energy and intensity of the precipitating electrons and protons (or, more precisely, their energetic tails), only a small part of the input energy goes into photonemission. The main part of the energy is released into collisions, plasma acceleration and heating, which subsequently result in a change of the entire conducting layer and in return energy/mass back to the magnetosphere. In-situ observations on board the Demeter satellite in the upper ionosphere allow to study the plasma variations in the regions of the field-aligned currents and sub-auroral polarisation stream.

Simultaneous measurements by Demeter and IMAGE demonstrate that the observed current variations are caused by spatial structuring of the source region and are not temporal or propagation effects. Thus, the field-aligned current region is revealed to comprise few layers of anti-parallel currents. Enhanced $\mathrm{O}^{+}$production inside the regions of the electron precipitation creates the plasma pressure gradients that causes the ions to move away from the overdense layers, i.e. first, perpendicular to the current sheet boundaries and then along and upward. This scenario is illustrated by a schematic drawing presented in Fig. 9. Temperature of outflowing $\mathrm{O}^{+}$is found to be colder than that of the ambient plasma.

Hiss emissions, which are regularly observed in the regions of the intense field-aligned currents with the source located inside the layers of the downward current, give the indications about the upward motion of the secondary electron population, with velocities higher than the electron thermal speed, i.e. $\sim 300 \mathrm{~km} \mathrm{~s}^{-1}$. Generation of these emissions in the ionosphere is a distinguished signature of intense electron exchange between the magnetosphere and ionosphere. 
Observed in the region of sub-auroral polarisation stream, the suprathermal $\mathrm{O}^{+}$is produced inside the SAPS boundary layer, which is characterised by strong sheared velocities. The suprathermal population is heated up to a few $\mathrm{eV}$, i.e. temperatures which are typical for the plasmaspheric ions. Thus, we suggest that the reversal in plasma convection driven at sub-auroral latitudes by the ring current polarisation field can be considered as a potential source region of the $\mathrm{O}^{+}$ outflow from the ionosphere.

Acknowledgements. We thank the Demeter team for the high quality work. We want to thank J. Jasperse and our referees for helpful comments, questions and suggestions.

Topical Editor M. Pinnock thanks W. K. Peterson and K. Kauristie for their help in evaluating this paper.

\section{References}

Anderson, P. C., Carpenter, D. L., Tsuruda, K., Mukai, T., and Rich, F. J.: Multisatellite observations of rapid subauroral ion drifts (SAID), J. Geophys. Res., 106, 29 585-29 599, 2001.

André, M. and Yau, A.: Theories and observations of ion energization and outflow in the high latitude magnetosphere, Space Sci. Rev., 80, 27-48, 1997.

Berthelier, J.-J., Godefroy, M., Leblanc, F., Séran, E., et al.: IAP, the thermal plasma analyzer on Demeter, Planet. Space Sci., 487501, 2006a.

Berthelier, J.-J., Godefroy, M., Leblanc, F., et al.: ICE, the electric field experiment on Demeter, Planet. Space Sci., 54, 456-471, 2006b.

Galperin, Y. I., Ponomarov, Y. N., and Zosinova, A. G.: Direct measurements of ion drift velocity in the upper ionosphere during a magnetic storm, Cosmicheskie Issled., 11, 273-283, 1973.

Germany, G. A., Spann, J. F., Parks, G. K., Brittnacher, M. J., Elsen, R., Chen, L., Lummerzheim, D., and Rees, M. H.: Auroral Observations from the POLAR Ultraviolet Imager (UVI), AGU Monograph, "Encounter Between Global Observations and Models in the ISTP Era", edited by: Horwitz, J., Gallagher, D., and Peterson, B., 1998.

Gurnett, D. A., Shawhan, S. D., and Shaw, R. R.: Auroral hiss, $\mathrm{Z}$ mode radiation and auroral kilometric radiation in the polar magnetosphere: DE-1 observations, J. Geophys. Res., 88, 329340,1983

Hirahara, M., Horwitz, J. L., Moore, T. E., Germany, G. A., Spann, J. F., Peterson, W. K., Shelley, E. G., Chandler,M. O., Giles, B. L., Craven, P. D., Pollock, C. J., Gurnett, D. A., Pickett, J. S., Persoon, A. M., Scudder, J. D., Maynard, N. C., Mozer, F. S., Brittnacher, M. J., and Nagai, T.:Relationship of topside ionospheric ion outflows to auroral forms and precipitation, plasma waves and convection observed by Polar, J. Geophys. Res., 103, 17391-17 410, 1998.

Hultqvist, B., André, M., Christon, S. P., Paschmann, G., and Sibeck,D.G.: Contributions of different source and loss processes to the plasma content of the magnetosphere, Space Sci. Rev., 88, 355-372, 1999.
Lin, C. S., Burch, J. L., Shawhan, S. D., and Gurnett, D. A.:Correlation of auroral hiss and upward electron beams near the polar cusp, J. Geophys. Res., 89, 925-935, 1984.

Mende, S. B., Heetderks, H., Frey, H., et al.: Far ultraviolet imaging from the IMAGE spacecraft, Space Sci. Rev., 91, 243-270, 2000.

Oksavik, K.,Greenwald, R. A , Ruohoniemi, J. M., Hairston, M. R., Paxton, L. J., Baker, J. B. H., Gjerloev, J. W., and Barnes, R. J.: First observations of the temporal/spatial variation of the sub-auroral polarization stream from the SuperDARN Wallops HF radar, Geophys. Res. Lett., 33, L12104, doi:10.1029/2006GL026256, 2006.

Peterson, W. K., Abe, T., Fukunishi, H., Greffen,M. J., Hayakawa, H., Kasahara, Y., Kimura, I., Matsuoka, A., Mukai, T., Nagatsuma, T., Tsuruda, K., Whalen, B. A., and Yau, A. W.: On the sources of energization of molecular ions at ionospheric altitudes, J. Geophys. Res., 99, 23 257-23 274, 1994.

Séran, E.: Reconstruction of the ion plasma parameters from the current measurements: Mathematical tool, Ann. Geophys., 21, 1159-1166, 2003, http://www.ann-geophys.net/21/1159/2003/.

Southwood, D. J. and Wolf, R. A. : An assessment of the role of precipitation in magnetospheric convection, J. Geophys. Res., 83, 5227-5232, 1978

Tokar, R. L. and Gary, S. P.: Electrostatic hiss and the beam driven electron acoustic instability in the dayside polar cusp, Geophys. Res. Lett., 11, 1180-1183, 1984.

Torr, M. R., Walker, J. C. G., and Torr, D. G.: Escape of fast oxygen from the atmosphere during geomagnetic storm, J. Geophys. Res., 79, 5267-5271, 1974.

Torr, M. R., Torr, D. G., Zukic, M., Johnson, R. B., Ajello, J., Banks, P., Clark, K., Cole, K., Keffer, C., Parks, G., Tsurutani, B., and Spann, J.: A far ultraviolet imager for the International Solar-Terrestrial Physics mission, Space Sci. Rev., 71, 329-383, 1995.

Walhund, J. E. and Opgenoorth, H. J.: EISCAT observation of strong ion flows from the F-region ionosphere during auroral activity, in: Preliminary results, Geophys. Res. Lett., 16, 727-730, 1989.

Wilson, G. R., Ober, D. M., Germany, G. A., and Lund, E. J.: The relationship between suprathermal heavy ion outflow and auroral electron energy deposition: Polar/UVI and FAST, J. Geophys. Res., 106, 18 981-18 994, 2001.

Yamamoto, T., Kaneda, E., Hayakawa, H., Mukai, T., Matsuoka, A., Machida, S., Fukunish, H., Kaya, N., Tsuruda, K., and Nishida, A.: Meridional structures of electric potentials relevant to premidnight discrete auroras: A case study from Akebono measurements, J. Geophys. Res., 98, 11 135-11 151, 1993.

Yau, A. W., Peterson, W. K., and Shelley, E. G.: Quantitative parametrization of energetic ionospheric ion outflow, in: Modeling of Magnetospheric Plasma, edited by: Moore, T. E. and Waite, J., Geophysics Monograph Series, 44, AGU, 211-217, 1988.

Yeh, H.-C. and Foster, J. C.: Storm time ion outflow at mid-latitude, J. Geophys. Res., 95, 7881-7891, 1990. 